\title{
Comportamento dos Zeros de Polinômios Sujeitos a uma Perturbação do Coeficiente Dominante
}

V.A. BOTTA P. ${ }^{1}$, M. MENEGUETTE Jr. ${ }^{2}$, Departamento de Matemática, Estatística e Computação, FCT, UNESP, 19060-900, Presidente Prudente, SP, Brasil.

Resumo. Em algumas áreas da Matemática, como na Análise Numérica, por exemplo, é de fundamental importância o comportamento dos zeros dos polinômios algébricos na análise de algumas questões. Considerando uma classe de polinômios cujos coeficientes satisfazem algumas condições, estudaremos o comportamento dos zeros desses polinômios na tentativa de encontrar um contra-exemplo para uma conjectura apresentada por Meneguette [5].

Palavras-chave. Zeros de polinômios, Disco unitário, Perturbação do coeficiente dominante.

\section{Introdução}

O comportamento dos zeros dos polinômios algébricos é uma subárea clássica da Análise que possui muitas questões a serem pesquisadas. Como exemplo, temos a seguinte conjectura, que é um problema que encontra-se em aberto em Meneguette [5]:

Conjectura 1.1. Sejam $P(z)=\sum_{i=0}^{n} a_{i} z^{i}$ um polinômio tal que

$$
0<a_{0} \leq a_{1} \leq \ldots \leq a_{n-1} \text { e } a_{n}<a_{n-1}
$$

cujos zeros encontram-se no disco unitário, e $P^{\prime}(z)$ com os coeficientes ordenados, isto é,

$$
0<a_{1} \leq 2 a_{2} \leq \ldots \leq(n-1) a_{n-1} \leq n a_{n} .
$$

Então, os zeros do polinômio

$$
P_{\gamma}(z)=\left(a_{n}+\gamma\right) z^{n}+a_{n-1} z^{n-1}+\ldots+a_{0}
$$

encontram-se no disco unitário, para todo $\gamma>0$.

\footnotetext{
${ }^{1}$ botta@fct.unesp.br

2 messias@fct.unesp.br
}

Recebido em 04 Setembro 2009; Aceito em 04 Maio 2010. 
Até o presente momento, o autor dessa conjectura não obteve respostas a respeito de sua validade ou não no caso geral. Botta, Meneguette e Cuminato [2] provaram sua validade no caso em que o polinômio $P(z)$ é um polinômio reflexivo, ou seja,

$$
a_{i}=a_{n-i}, a_{i}>0, i=0,1,2, \ldots, n .
$$

Além disso, Botta [3] mostrou sua validade quando os coeficientes do polinômio $P(z)$ apresentado na conjectura satisfazem as seguintes condições:

$$
0<a_{0}<a_{1}<\ldots<a_{n-1}>a_{n}
$$

e

$$
p a_{i}<(p-1) a_{i+1}, i=0,1, \ldots, n-2, \text { e } p a_{n}>(p-1) a_{n-1},
$$

onde $p$ é um número inteiro positivo, $p>1$.

$\mathrm{Na}$ tentativa de buscar um contra-exemplo para a Conjectura 1.1, analisaremos, nesse trabalho, uma classe de polinômios $P(z)=\sum_{i=0}^{n} a_{i} z^{i}$ cujos coeficientes, para $n=k m-1$ e $k, m \in \mathbb{N}$, satisfazem

$$
\begin{aligned}
0<a_{0}=\ldots=a_{m-1} & <a_{m}=\ldots=a_{2 m-1}<a_{2 m}=\ldots=a_{3 m-1}<\ldots \\
& <a_{(k-1) m}=\ldots=a_{k m-1}=a_{n}
\end{aligned}
$$

ou seja, os coeficientes de $P(z)$ são iguais para cada grupo de $m$ elementos consecutivos.

Como $P(z)$ tem os coeficientes ordenados, pelo Teorema de Eneström-Kakeya, que será apresentado a seguir, temos que os zeros de $P(z)$ encontram-se em $|z| \leq 1$. Além disso, pelo menos um zero de $P(z)$ tem módulo um. Portanto, qualquer perturbação no coeficiente dominante de $P(z)$ acarretará uma perturbação nos zeros de $P(z)$, podendo estes entrarem ou saírem do disco unitário quando o parâmetro $\gamma$ cresce. Experimentos computacionais revelam uma tendência dos zeros saírem do disco unitário. Então, uma questão que surge é a seguinte: sendo $P_{\lambda}(z)=$ $\sum_{i=0}^{n-1} a_{i} z^{i}+\left(a_{n}-\lambda\right) z^{n}$, será possível os zeros de $P_{\lambda}(z)$ voltarem a entrar no disco unitário dependendo de $\lambda$ ? Se isso ocorrer, teríamos um contra-exemplo para a Conjectura 1.1, pois assim existiria $\gamma>0$ tal que os zeros de $P_{\gamma}(z)$ definido na Conjectura 1.1 poderiam sair do disco unitário, contradizendo a tese da Conjectura 1.1. Portanto, para responder a essa questão, apresentaremos, no decorrer desse trabalho, uma análise do comportamento dos zeros de $P_{\lambda}(z)$.

\section{Resultados Clássicos}

O primeiro resultado a ser apresentado é o Teorema de Eneström-Kakeya, que determina a quantidade de zeros de um polinômio no disco unitário. 
Teorema 2.1 (Eneström-Kakeya). Seja $P(z)=\sum_{i=0}^{n} a_{i} z^{i}$ um polinômio cujos coeficientes $a_{i}, i=0,1, \ldots, n$, satisfazem $0<a_{0} \leq a_{1} \leq \ldots \leq a_{n}$. Então, $P(z)$ possui seus zeros em $|z| \leq 1$.

Um resultado mais amplo e equivalente ao Teorema de Eneström-Kakeya, que pode ser encontrado em Anderson, Saff e Varga [1], é o seguinte:

Teorema 2.2. O polinômio $P(z)=\sum_{i=0}^{n} a_{i} z^{i}, a_{i}>0, i=0,1, \ldots, n$, tem todos os seus zeros na região $\alpha \leq|z| \leq \beta$, onde $\alpha=\min _{0 \leq i \leq n-1}\left\{\frac{a_{i}}{a_{i+1}}\right\}$ e $\beta=\max _{0 \leq i \leq n-1}\left\{\frac{a_{i}}{a_{i+1}}\right\}$.

Consideremos

$$
\begin{gathered}
\bar{S}=\left\{j=1, \ldots, n+1: \beta a_{n+1-j}-a_{n-j}>0\right\}, \text { onde } a_{-1}:=0, \\
\underline{S}=\left\{j=1, \ldots, n+1: a_{j-1}-\alpha a_{j}>0\right\}, \text { onde } a_{n+1}:=0, \\
\bar{k}=\operatorname{mdc}\{j: j \in \bar{S}\} \text { e } \underline{k}=\operatorname{mdc}\{j: j \in \underline{S}\},
\end{gathered}
$$

onde $m d c$ é o máximo divisor comum.

Teorema 2.3. Seja $P(z)=\sum_{i=0}^{n} a_{i} z^{i}, a_{i}>0, i=0,1, \ldots, n$. Todos os zeros de $P(z)$ encontram-se na região $\alpha \leq|z| \leq \beta$. Além disso, $P(z)$ pode se anular em $|z|=\beta$ se, e somente se, $\bar{k}>1$. Se $\bar{k}>1$, os zeros de $P(z)$ em $|z|=\beta$ são simples $e$ dados por

$$
\left\{\beta e^{2 \pi i j / \bar{k}}, j=1, \ldots, \bar{k}-1\right\}
$$

Da mesma forma, $P(z)$ pode se anular em $|z|=\alpha$ se, e somente se, $\underline{k}>1$. Se $\underline{k}>1$, os zeros de $P(z)$ em $|z|=\alpha$ são simples $e$ dados por

$$
\left\{\alpha e^{2 \pi i j / \underline{k}}, j=1, \ldots, \underline{k}-1\right\}
$$
[1].

A demonstração desse resultado pode ser encontrada em Anderson, Saff e Varga

O resultado a seguir mostra que se um polinômio $P(z)$ tem coeficientes $a_{i}$, $i=0,1, \ldots, n$, satisfazendo (1.1), então $P(z)$ tem $m-1$ zeros simples de módulo um.

Teorema 2.4. O polinômio $P(z)=\sum_{i=0}^{n} a_{i} z^{i}$ tal que seus coeficientes satisfazem (1.1) possui $m-1$ zeros simples de módulo um.

Demonstração. Como $P(z)$ satisfaz (1.1), podemos escrevê-lo da forma

$$
P(z)=\left(1+z+\ldots+z^{m-1}\right)\left(a_{0}+a_{m} z^{m}+\ldots+a_{(k-1) m} z^{(k-1) m}\right)=Q(z) R(z) .
$$

Para o polinômio $Q(z)$, temos que $\alpha=\beta=1$. Logo, pelo teorema anterior, todos os seus $m-1$ zeros estão em $|z|=1$ e, além disso, como $\bar{k}=m$, são simples. 
Utilizaremos também uma das fórmulas de Vieta, dada por

$$
z_{1} z_{2} \ldots z_{n}=(-1)^{n} \frac{a_{0}}{a_{n}}
$$

onde $z_{1}, z_{2}, \ldots, z_{n}$ são os zeros do polinômio $P(z)=\sum_{i=0}^{n} a_{i} z^{i}$.

Suponhamos que o polinômio $P(z)$ tenha $p(p \leq n)$ zeros em $|z| \leq 1$.

Associado a ele será considerado o polinômio

$$
P^{*}(z)=z^{n} P\left(\frac{1}{z}\right)=\sum_{i=0}^{n} a_{i} z^{n-i}=a_{0} \prod_{j=1}^{n}\left(z-z_{j}^{*}\right),
$$

sendo seus zeros $z_{k}^{*}$, em relação ao disco unitário, os inversos dos zeros $z_{k}$ de $P(z)$, isto é, $z_{k}^{*}=\frac{1}{z_{k}}$.

Logo, qualquer zero de $P(z)$ que possui módulo um é também zero de $P^{*}(z)$ e se $P(z)$ não tem zeros em $|z|=1, P^{*}(z)$ também não tem zeros em $|z|=1$.

Além disso, se $P(z)$ não tem zeros em $|z|=1$ e $p$ zeros em $|z|<1$, então $P^{*}(z)$ possui $n-p$ zeros em $|z|<1$.

Seja, agora, a sequência de polinômios

$$
P_{j}(z)=\sum_{k=0}^{n-j} a_{k}^{(j)} z^{k}
$$

onde $P_{0}(z)=P(z)$ e

$$
P_{j+1}(z)=a_{0}^{(j)} P_{j}(z)-a_{n-j}^{(j)} P_{j}^{*}(z), j=0, \ldots, n-1 .
$$

Então,

$$
a_{k}^{(j+1)}=a_{0}^{(j)} a_{k}^{(j)}-a_{n-j}^{(j)} a_{n-j-k}^{(j)}
$$

Se em cada polinômio $P_{j}(z)$ o termo constante $a_{0}^{(j)}$ é representado por $\delta_{j}$, então

$$
\delta_{j+1}=\left|a_{0}^{(j)}\right|^{2}-\left|a_{n-j}^{(j)}\right|^{2}=a_{0}^{(j+1)}, j=0, \ldots, n-1 .
$$

Lema 2.1. Considerando $a_{0} \neq 0$, se $P_{j}$ tem $p_{j}$ zeros em $|z| \leq 1$ e se $\delta_{j+1} \neq 0$, então $P_{j+1}$ tem

$$
p_{j+1}=\frac{1}{2}\left\{n-j-\left[(n-j)-2 p_{j}\right] \operatorname{sinal}\left(\delta_{j+1}\right)\right\}
$$

zeros em $|z| \leq 1$. Além disso, $P_{j+1}$ tem os mesmos zeros em $|z|=1$ que $P_{j}$.

A demonstração deste lema pode ser encontrada em Marden [4].

Teorema 2.5. Se $\left|a_{0}\right|<\left|a_{n}\right|, a_{0} \neq 0, P(z)$ tem todos os seus zeros no disco unitário se, e somente se, $P_{1}^{*}(z)$ tem também todos os seus zeros em $|z| \leq 1$.

A demonstração deste resultado segue do Lema 2.1 e mais detalhes podem ser encontrados em Schur [6], [7]. 


\section{Análise dos Resultados}

Sejam

$$
P_{\lambda}(z)=a_{0}+a_{1} z+\ldots+a_{n-1} z^{n-1}+\left(a_{n}-\lambda\right) z^{n}, \lambda>0
$$

$\mathrm{e}$

$$
P(z)=a_{0}+a_{1} z+\ldots+a_{n-1} z^{n-1}+a_{n} z^{n}
$$

cujos coeficientes $a_{i}, i=0,1, \ldots, n$, satisfazem (1.1).

Vamos considerar $\lambda \leq a_{n}-a_{0}$, pois se $\lambda>a_{n}-a_{0}$ pela fórmula (2.1) já segue que $P_{\lambda}(z)$ possui pelo menos um zero fora do disco unitário.

Seja, primeiramente, o caso em que $a_{0}=a_{1}=\ldots=a_{n}$. Então, para $\lambda>0$ e tal que $a_{n}-\lambda>0$, temos que pelo menos um zero de $P_{\lambda}(z)$ encontra-se fora do disco unitário, pois

$$
a_{0}=a_{n} \Rightarrow a_{0}>a_{n}-\lambda \Rightarrow\left|a_{0}\right|>\left|a_{n}-\lambda\right|
$$

e, pela expressão $(2.1)$

$$
\left|z_{1}\right|\left|z_{2}\right| \ldots\left|z_{n}\right|=\frac{\left|a_{0}\right|}{\left|a_{n}-\lambda\right|}>1
$$

onde $z_{1}, z_{2}, \ldots, z_{n}$ são os zeros de $P_{\lambda}(z)$, indicando que pelo menos um zero de $P_{\lambda}(z)$ encontra-se fora do disco unitário. Portanto, se $a_{0}=a_{1}=\ldots=a_{n}$, não foi possível encontrar um contra-exemplo para a conjectura.

Analisaremos agora os seguintes casos:

1. $m$ é par e $n$ é ímpar:

Observe que

$$
P(-1)=a_{0}-a_{1}+a_{2}-a_{3}+\ldots+a_{n-1}-a_{n}=0,
$$

ou seja, $z=-1$ é um zero de $P(z)$.

Além disso,

$$
P_{\lambda}(-1)=P(-1)+\lambda=\lambda>0 \text {. }
$$

Como, para $z \in \mathbb{R}^{-}, \lim _{z \rightarrow-\infty} P_{\lambda}(z)<0$ e $P_{\lambda}(-1)>0$, então existe pelo menos um zero real de $P_{\lambda}(z)$ em $(-\infty,-1)$, ou seja, pelo menos um zero encontra-se fora do disco unitário. Portanto, com essas condições sobre os coeficientes, não existe um contra-exemplo para a Conjectura 1.1.

2. $m$ e $n$ são pares:

Este caso é impossível de ocorrer, pois como $n$ é par, o polinômio $P_{\lambda}(z)$ tem um número ímpar de coeficientes, sendo impossível agrupá-los em conjuntos com $m$ coeficientes, pois $m$ é par.

3. Analisaremos ao mesmo tempo os casos em que $m$ é ímpar e $n$ é par e $m$ e $n$ são ímpares: 
Considerando a expressão $(2.2)$ para o polinômio $P_{\lambda}(z)$, temos que

$$
\begin{aligned}
a_{0, \lambda}^{(1)} & =a_{0}^{2}-\left(a_{n}-\lambda\right)^{2}<0 \\
a_{n-1, \lambda}^{(1)} & =a_{0} a_{n-1}-a_{1}\left(a_{n}-\lambda\right)=\lambda a_{1}>0 .
\end{aligned}
$$

Então,

$$
\left|a_{0, \lambda}^{(1)}\right|-\left|a_{n-1, \lambda}^{(1)}\right|=\lambda^{2}-\left(a_{1}+2 a_{n}\right) \lambda+a_{n}^{2}-a_{0}^{2} .
$$

Resolvendo a equação $\lambda^{2}-\left(a_{1}+2 a_{n}\right) \lambda+a_{n}^{2}-a_{0}^{2}=0$, segue que

$$
\lambda=\frac{a_{1}+2 a_{n} \pm \sqrt{a_{0}\left(4 a_{n}+5 a_{0}\right)}}{2} .
$$

Consideremos $\lambda_{1}=\frac{a_{0}+2 a_{n}-\sqrt{a_{0}\left(4 a_{n}+5 a_{0}\right)}}{2}>0$ (observe que $\left.a_{0}+2 a_{n}>\sqrt{a_{0}\left(4 a_{n}+5 a_{0}\right)}\right)$ e $\lambda_{2}=\frac{a_{0}+2 a_{n}+\sqrt{a_{0}\left(4 a_{n}+5 a_{0}\right)}}{2}>0$ os zeros reais de (3.1).

Para $\lambda_{1}<\lambda<\lambda_{2}$, segue que $\left|a_{0, \lambda}^{(1)}\right|-\left|a_{n-1, \lambda}^{(1)}\right|<0$, ou seja, $\left|a_{0, \lambda}^{(1)}\right|<\left|a_{n-1, \lambda}^{(1)}\right|$, o que implica que o polinômio $P_{1, \gamma}^{*}(z)$ tem zeros fora do disco unitário, pois considerando $w_{1}, w_{2}, \ldots, w_{n-1}$ os zeros de $P_{1, \gamma}^{*}(z)=a_{0, \gamma}^{(1)} z^{n-1}+\ldots+a_{n-1, \gamma}^{(1)}$, temos, de (2.1),

$$
\left|w_{1}\right|\left|w_{2}\right| \ldots\left|w_{n-1}\right|=\frac{\left|a_{n-1, \gamma}^{(1)}\right|}{\left|a_{0, \gamma}^{(1)}\right|}>1 .
$$

Logo, pelo Teorema 2.5, $P_{\lambda}(z)$ tem zeros fora do disco unitário.

Mas $\lambda_{2}>a_{n}-a_{0}$. Portanto, é conveniente considerarmos o intervalo $\lambda_{1}<\lambda<a_{n}-a_{0}$. Logo, para $\lambda_{1}<\lambda<a_{n}-a_{0}, P_{\lambda}(z)$ tem zeros fora do disco unitário.

Analiticamente, para $0<\lambda \leq \lambda_{1}$, onde $\left|a_{0, \lambda}^{(1)}\right|-\left|a_{n-1, \lambda}^{(1)}\right| \geq 0$, os cálculos mostraram-se complicados, sendo necessário um estudo mais complexo e detalhado do assunto, que não será abordado nesse trabalho. Mas, através de simulações numéricas, é possível verificar que os zeros de $P_{\lambda}(z)$, para $0<\lambda \leq \lambda_{1}$, encontram-se fora do disco unitário, como podemos observar nos Exemplos 4.4 e 4.5 da próxima seção.

\section{Exemplos}

Nessa seção apresentaremos alguns exemplos de polinômios cujos coeficientes satisfazem as condições apresentadas na seção anterior. Nas figuras seguintes mostraremos o comportamento dos zeros do polinômio $P_{\lambda}(z)$ (representados pelos pontos) para $\lambda$ real, $\lambda>0$. Os zeros de $P(z)$ estão representados pelos pontos maiores e mais escuros. À medida que $\lambda$ aumenta, os pontos nas figuras que representam os zeros de $P_{\lambda}(z)$ vão diminuindo e tornando-se mais claros. 
Conjectura 4.1. Seja

$$
P(z)=z^{4}+z^{3}+z^{2}+z+1,
$$

cujos zeros encontram-se em $|z|=1$.

Nesse caso, o polinômio

$$
P_{\lambda}(z)=(1-\lambda) z^{4}+z^{3}+z^{2}+z+1
$$

possui zeros fora do disco unitário, como podemos observar na Figura 1.

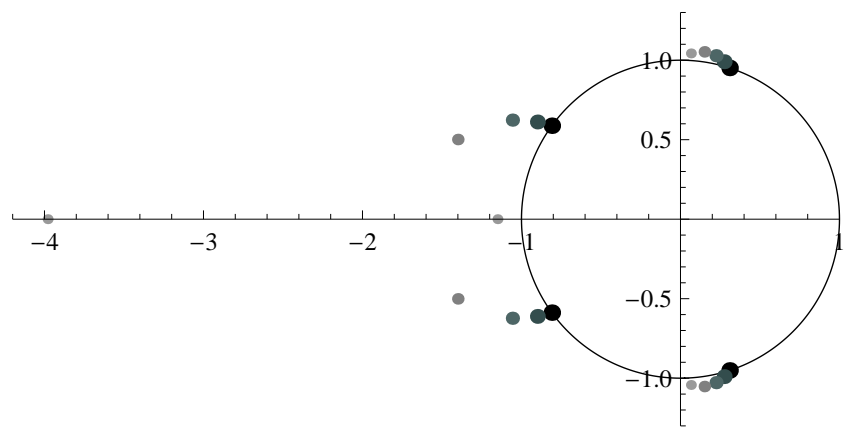

Figura 1: Comportamento dos zeros de $P_{\lambda}(z)=(1-\lambda) z^{4}+z^{3}+z^{2}+z+1, \lambda=0,0.2$, $0.4,0.6$ e 0.8 .

Conjectura 4.2. Consideremos

$$
P(z)=3 z^{3}+3 z^{2}+z+1
$$

cujos zeros encontram-se em $|z| \leq 1$.

Observe que

$$
P_{\lambda}(z)=(3-\lambda) z^{3}+3 z^{2}+z+1 .
$$

O polinômio $P_{\lambda}(z)$ possui zeros fora do disco (zero real negativo), como podemos ver na Figura 2. Além disso, conforme análise do item 1 da seção anterior (caso em que $m$ é par e $n$ é ímpar), temos a garantia de que $P_{\lambda}(z)$ possui zeros fora do disco unitário.

Conjectura 4.3. Seja

$$
P(z)=2 z^{7}+2 z^{6}+2 z^{5}+2 z^{4}+z^{3}+z^{2}+z+1,
$$

cujos zeros encontram-se em $|z| \leq 1$.

O polinômio

$$
P_{\lambda}(z)=(2-\lambda) z^{7}+2 z^{6}+2 z^{5}+2 z^{4}+z^{3}+z^{2}+z+1
$$

possui zeros fora do disco unitário (zero real negativo), conforme podemos ver na Figura 3 e cujo resultado está garantido pela análise feita na seção anterior para o caso em que $m$ é par e $n$ é ímpar. 


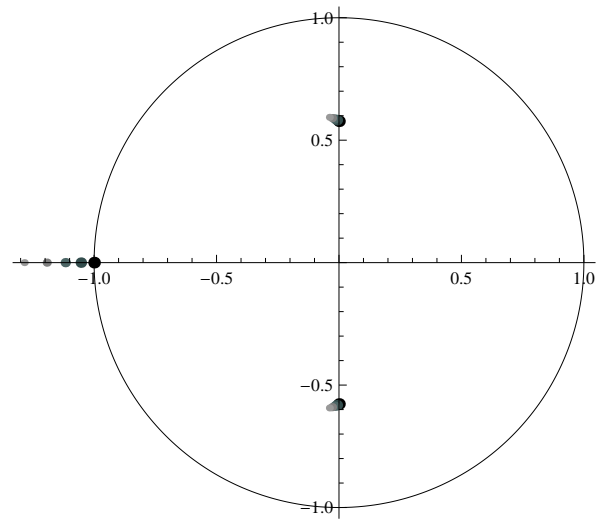

Figura 2: Comportamento dos zeros de $P_{\lambda}(z)=(3-\lambda) z^{3}+3 z^{2}+z+1, \lambda=0,0.2,0.4$, 0.6 e 0.8 .

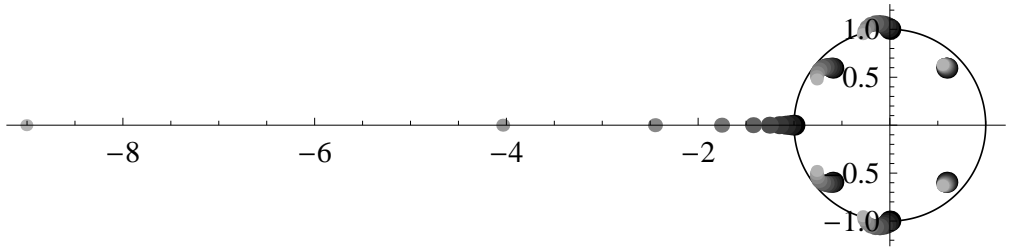

Figura 3: Comportamento dos zeros de $P_{\lambda}(z)=(2-\lambda) z^{7}+2 z^{6}+2 z^{5}+2 z^{4}+z^{3}+z^{2}+z+1$, $\lambda=0,0.2,0.4,0.6, \ldots, 1.8$.

\section{Conjectura 4.4. O polinômio}

$$
P(z)=7 z^{8}+7 z^{7}+7 z^{6}+4 z^{5}+4 z^{4}+4 z^{3}+z^{2}+z+1,
$$

possui seus zeros em $|z| \leq 1$.

O polinômio

$$
P_{\lambda}(z)=(7-\lambda) z^{8}+7 z^{7}+7 z^{6}+4 z^{5}+4 z^{4}+4 z^{3}+z^{2}+z+1
$$

possui zeros fora do disco, como podemos observar na Figura 4.

Pelo resultado encontrado na seção anterior (caso em que $m$ é ímpar e $n$ é par), temos a garantia de que os zeros de $P_{\lambda}(z)$ encontram-se fora do disco unitário para $4.6<\lambda<6$ (para $\lambda>6$, o resultado está garantido pela fórmula de Vieta). Para $0<\lambda \leq 4.6$, através da Figura 4 podemos observar que $P_{\lambda}(z)$ possui zeros fora do disco unitário, mas a análise algébrica desse caso será objeto de estudos futuros.

Conjectura 4.5. Seja

$$
P(z)=10 z^{11}+10 z^{10}+10 z^{9}+6 z^{8}+6 z^{7}+6 z^{6}+3 z^{5}+3 z^{4}+3 z^{3}+z^{2}+z+1,
$$

cujos zeros encontram-se em $|z| \leq 1$. 


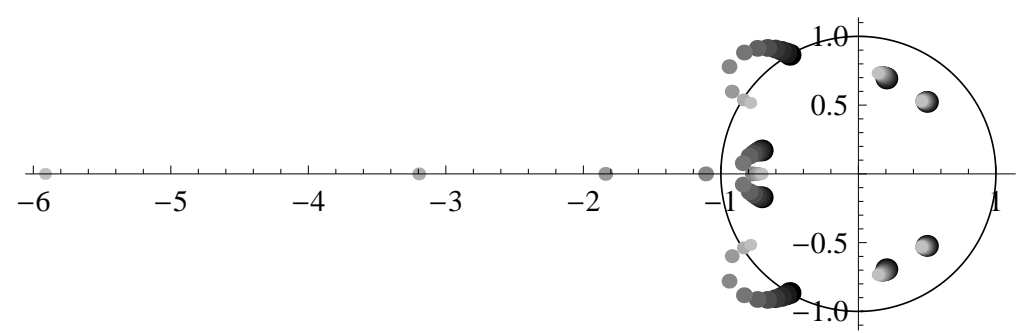

Figura 4: Comportamento dos zeros de $P_{\lambda}(z)=(7-\lambda) z^{8}+7 z^{7}+7 z^{6}+4 z^{5}+4 z^{4}+4 z^{3}+$ $z^{2}+z+1, \lambda=0,0.6,1.2,1.8, \ldots, 6$.

O polinômio

$P_{\lambda}(z)=(10-\lambda) z^{11}+10 z^{10}+10 z^{9}+6 z^{8}+6 z^{7}+6 z^{6}+3 z^{5}+3 z^{4}+3 z^{3}+z^{2}+z+1$

possui zeros fora do disco, como podemos observar na Figura 5. Nesse caso, $m$ e $n$ são ímpares. Então, temos a garantia de que os zeros de $P_{\lambda}(z)$ encontram-se fora do disco unitário para $\lambda_{1}=7.1<\lambda<9$ (para $\lambda>9$, o resultado está garantido pela fórmula de Vieta). A análise algébrica para o caso em que $0<\lambda \leq 7.1$ será estudada futuramente.

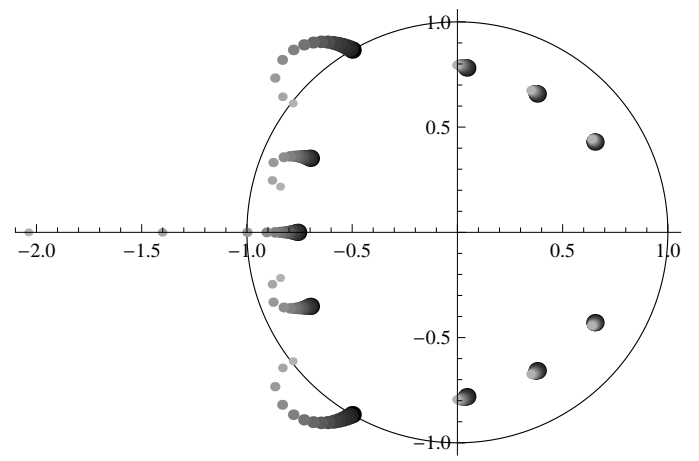

Figura 5: Comportamento dos zeros de $P_{\lambda}(z)=(10-\lambda) z^{11}+10 z^{10}+10 z^{9}+6 z^{8}+6 z^{7}+$ $6 z^{6}+3 z^{5}+3 z^{4}+3 z^{3}+z^{2}+z+1, \lambda=0,0.5,1.0,1.5, \ldots, 7$.

\section{Conclusão}

Analisamos, nesse trabalho, o comportamento dos zeros do polinômio $P_{\lambda}(z)$ cujos coeficientes satisfazem as condições apresentadas na Seção 3 . No caso em que $a_{0}=$ $a_{1}=\ldots=a_{n}$, pelo menos um zero de $P_{\lambda}(z)$ encontra-se fora do disco unitário. Quando $m$ é par e $n$ é ímpar, foi possível mostrar que sempre existirá um zero real negativo cujo módulo é maior que um. Não existirá o caso em que $m$ e $n$ são pares. O caso em que $m$ e $n$ são ímpares e $m$ é ímpar e $n$ é par foi verificado 
para $\lambda_{1}=\frac{a_{0}+2 a_{n}-\sqrt{a_{0}\left(4 a_{n}+5 a_{0}\right)}}{2}<\lambda<a_{n}-a_{0}$. Portanto, não foi possível encontrar um contra-exemplo para a Conjectura 1.1 nos casos citados, sendo cada vez mais fortes as evidências de que ela é verdadeira.

Abstract. In some areas of Mathematics, for example in Numerical Analysis, the behavior of perturbed polynomial zeros is relevant to answer some questions. Considering a class of polynomials under certain coefficients conditions, we study the perturbed zeros behavior in an attempt to find a counter-example to a conjecture by Meneguette [5].

\section{Referências}

[1] N. Anderson, E.B. Saff, R.S. Varga, On the Eneström-Kakeya theorem and its sharpness, Linear Algebra and Appl., 28 (1979), 5-16.

[2] V.A. Botta, M. Meneguette Jr., J.A. Cuminato, Sobre uma generalização do Teorema de Eneström-Kakeya: polinômio reflexivo, TEMA Tend. Mat. Apl. Comput., 9, No. 1 (2008), 125-132.

[3] V.A. Botta, "Zeros de Polinômios Característicos e Estabilidade de Métodos Numéricos", Tese de Doutorado, ICMC-USP, São Carlos, 2008.

[4] M. Marden, "Geometry of Polynomials", American Mathematical Society, Providence, RI, 1966.

[5] M. Meneguette Jr., Zeros in the unit disk, SIAM Review, 36 (1994), 656-657.

[6] I. Schur, Über Potenzreihen, die in Innern des Einheitskreises beschränkt sind, J. Reine Angew. Math., 147 (1917), 205-232.

[7] I. Schur, Über Polynome, die nur in Innern des Einheitkreis verschwinden, $J$. Reine Angew. Math., 148 (1918), 122-145. 